\title{
Timeline Improvement of Hierarchical Clustering Algorithms for Wireless Sensor Networks
}

\author{
Nassirah Laloo \\ University of Technology, Mauritius
}

\author{
Mohammad Sameer Sunhaloo \\ University of Technology, Mauritius
}

\author{
Raja K. Subramanian
}

\begin{abstract}
Wireless sensor networks have a wide range of practical and useful applications. Since a wireless sensor network is driven by batteries, energy consumption is a critical factor to determine the life of the network. The energy of the nodes are dispensed mainly through sensing, processing and communication. Energy efficiency is an area that has attracted much research in the field of wireless sensor networks. In view of prolonging the network lifetime, various hierarchical clustering algorithms have been proposed in literature. In this paper, an analysis of the timeline improvement of hierarchical cluster based protocols for wireless sensor networks is presented.
\end{abstract}

\section{General Terms}

Wireless Networks, Hierarchical Clustering

\section{Keywords}

Wireless sensor networks, network lifetime, hierarchical cluster based protocols

\section{INTRODUCTION}

A wireless sensor network (WSN) is made up of thousands to millions of tiny devices called sensor nodes, which collectively sense, process and communicate data about the region under focus. The battery-operated nodes are short lived consequently making energy a significant resource. Continuous research is being carried out to minimise the energy consumption of the sensor nodes. The nodes' energy are dispensed mainly through sensing, processing and communication. Since more energy is consumed in data transmission, it is important to design energy optimized routing algorithm to combine both energy efficiency and energy balancing in prolonging the network lifetime. Cluster based hierarchical protocols have proved to a great extent to be effective in energy management [1].

In hierarchical cluster based protocols, the two-tier communication is often considered between nodes in the WSN, to enhance the lifespan of the low energy battery powered nodes. The cluster head $(\mathrm{CH})$ nodes, often at the upper level are responsible for aggregation and forwarding of aggregated data to the base station (BS). The normal nodes, situated at the lower levels perform regular sensing and communication with their corresponding $\mathrm{CH}$ nodes.
The Low-Energy Adaptive Clustering Hierarchy (LEACH) algorithm is the first hierarchical clustering algorithm for wireless sensor networks and has since its inception, in 2000, been considered as the state of the art algorithm for many researches in the field [2]. While there are considerable ongoing research on hierarchical clustering, the LEACH algorithm is still referenced and compared to. This paper analyses the timeline improvement of hierarchical clustering protocol for Wireless Sensor Networks.

\section{THE LEACH ALGORITHM}

The LEACH is a self-organising and adaptive clustering protocol, which operates in two phases; namely, the set up phase and the steady phase. An outline of the operations per protocol round is given below.

\section{- Set-Up Phase}

-Advertisement Phase

- Independent decision of nodes to become $\mathrm{CH}$ nodes.

- $\mathrm{CH}$ nodes use advertisement (ADV) packet to inform neighbourhood nodes that they are $\mathrm{CH}$ nodes.

- Non-CH nodes select the ADV packet based on the strength of received signal. The strongest signal is selected.

- Cluster Set-Up Phase

- Normal member nodes forward join packet, using CarrierSense Multiple Access (CSMA) to inform the $\mathrm{CH}$ of their membership to particular cluster.

- In this phase, the $\mathrm{CH}$ knows the number of member nodes and IDs. The $\mathrm{CH}$ creates a Time Division Multiple Access (TDMA) schedule and forward to cluster members.

- Steady Phase

-Data transmission takes place. Member nodes forward data during their allocated TDMA slot to $\mathrm{CH}$

- Radio of non- $\mathrm{CH}$ nodes is turned off until the nodes are allocated TDMA.

-Once all data collected, the $\mathrm{CH}$ nodes aggregates the data and send to the BS.

In the set up phase the nodes organise themselves into clusters, with one node acting as the $\mathrm{CH}$. These $\mathrm{CH}$ nodes accept data from local cluster nodes, aggregate the data and forward the aggregated data to the BS. The election of the $\mathrm{CH}$ is done stochastically in each round. In the second phase, the data is forwarded to the BS. Fig 1 illustrates the working of the LEACH protocol for one round. LEACH adopts a distributed cluster formation process. The nodes 


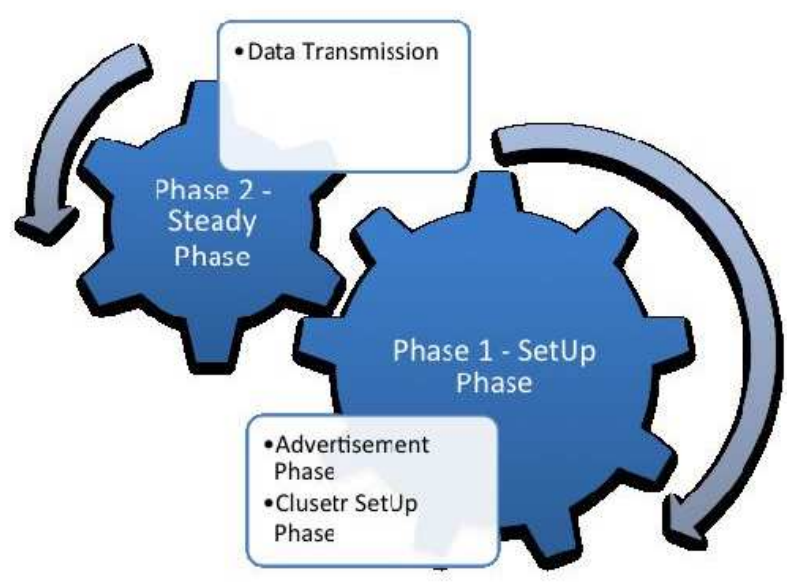

Fig. 1. Phases in LEACH

elect themselves as $\mathrm{CH}$ based on the equation [2]

$$
P_{i}(t)= \begin{cases}k /(N-k(r \bmod N / k)), & C_{i}(t)=1 ; \\ 0, & C_{i}(t)=0 .\end{cases}
$$

where $r$ is the number of rounds that have passed and $C_{i}(t)=0$ if node $i$ has already been a cluster head in the most recent $(r \bmod N / k)$ rounds and 1 otherwise. Election and formation of the $\mathrm{CH}$ is done without knowing the exact location of any nodes in the network. Thus no global communication is required to set up the cluster.

The $\mathrm{CH}$ nodes inform all the other nodes in the network of their respective status by broadcasting an ADV message using CSMA MAC protocol. The TDMA schedule is created and forwarded to the normal cluster nodes. The $\mathrm{CH}$ nodes use high power signal to send the ADV message to ensure that all the nodes are reached.

The non- $\mathrm{CH}$ nodes choose which $\mathrm{CH}$ to join based on the received signal strength of the ADV. The non- $\mathrm{CH}$ nodes join the $\mathrm{CH}$, which requires the minimum communication energy. Assuming a symmetric propagation channel, the $\mathrm{CH}$ heard with the largest signal strength ID is the $\mathrm{CH}$ to whom minimum amount of transmission energy is needed for communication. After each normal node decides to which cluster it belongs, it informs the $\mathrm{CH}$ node by sending a JOIN request message (JOIN-REQ) using CSMA MAC protocol. To avoid the hidden terminal problem the normal node uses large power to send the JOIN-REQ message.

After the network is divided into clusters, the $\mathrm{CH}$ creates TDMA time slots for its member nodes. The $\mathrm{CH}$ nodes also chose a CDMA code for ultimate data transmission to the BS.

In the steady phase, the sensor nodes sense and forward data to their respective $\mathrm{CH}$ within their allocated TDMA time slot. The $\mathrm{CH}$ must keep the receiver on to receive all data from nodes in the cluster. Once all data is received it operates on the data by performing data aggregation and forwarding the resulting data to the BS. Since the $\mathrm{BS}$ is often far and the data message is large, high-energy transmission is required. To reduce intra cluster interference, each cluster communicates using Direct Sequence Spread Spectrum (DSSS), whereby they use a unique spreading code for communication. Nodes communication is based on transmitter based code assignment. Thus all nodes in the cluster transmit data to the $\mathrm{CH}$ using the spreading code and the $\mathrm{CH}$ filters all received energy using this code. The data from the $\mathrm{CH}$ node to the $\mathrm{BS}$ is sent using fixed spreading code and CSMA approach.

\subsection{Limitations of LEACH}

Although LEACH is still emphasised in recent research works and its contributions remain unquestioned, there are a number of limitations which are incurred with the protocol. The protocol operates in rounds, each with two phases. The set up phase causes overhead since $\mathrm{CH}$ nodes need to be elected in each round. Moreover, the $\mathrm{CH}$ nodes are selected at random with no optimisation on the $\mathrm{CH}$ distribution. The low energy nodes are considered together with highenergy nodes on the election of the $\mathrm{CH}$ node. In $\mathrm{LEACH}$, the $\mathrm{CH}$ uses single-hop for communication to the base station. As a result the $\mathrm{LEACH}$ protocol put much strain on its $\mathrm{CH}$ and the network is as a result not scalable. These limitations have triggered different studies in the field of hierarchical clustering.

\section{IMPROVEMENT UPON THE LEACH ALGORITHM}

The LEACH algorithm has over the years been improved in several ways. Improvement of LEACH exists as family of LEACH whereby each improved algorithm is a small improvement upon the LEACH algorithm. T-LEACH [3], E-LEACH [4], M-LEACH [4], B4-LEACH [5], V-LEACH [6] amongst others form part of the LEACH family as depicted in Fig 2 Other algorithms are mod-

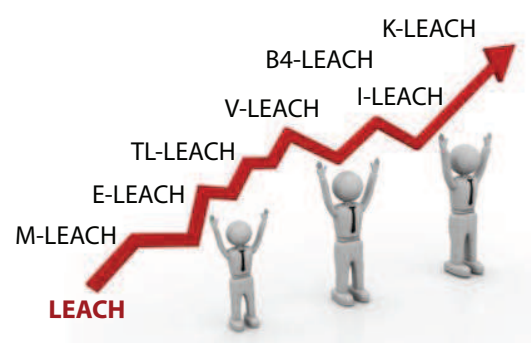

Fig. 2. Family of LEACH

ified versions of the LEACH algorithm with added concepts and consequently forming different families, as shown in Fig 3 PEGASIS [7], H-PEGASIS [8] and EB-PEGASIS [9] are a family group of such improvements referred to as the PEGASIS family. Similarly TEEN [10] and APTEEN [11] are improved LEACH algorithms falling in a different family group.

Madheswaran \& Shanmugasundaram [12] worked on the enhancements of the LEACH algorithm. They classified LEACH enhancement algorithms according to the following three categories: Modified $\mathrm{CH}$ selection algorithm, Energy Aware Algorithms, and Optimisation in $\mathrm{CH}$ Selection. Gnanambigai et al. [13] in their work made a comparison of nine descendants of LEACH on different metrics. Dhawan \& Waraich [14] made a comparative study on LEACH routing protocol and its variants and conclude with the need to improve towards more robust, reliable and efficient network. Deshmukh \& Patil [15] made a review of LEACH descendants to show their improved performance in terms 


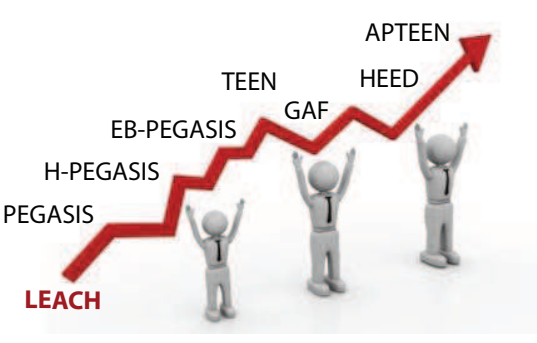

Fig. 3. Other Hierarchical Protocols

of network scalability, network stability, network security, energy efficiency and network lifetime.

To the best of our knowledge, work that evaluates LEACH evolution, compare only LEACH family that are improved by slight modifications to LEACH. None of the work considered related families of protocols that are based on LEACH with an added concept to create a different family.

\subsection{Family of LEACH}

In this section, different existing modifiers of LEACH are listed and the corresponding improvements brought to LEACH are highlighted.

Year 2016

- LEACH-MAC [16] improves upon the dynamic, distributed and randomised (DDR) algorithms for clustering. LEACH-MAC controls the randomness of generated number of $\mathrm{CH}$ nodes.

Year 2014

- O-LEACH [17] improves cluster selection by dynamically selecting cluster based on the residual energy of nodes.

- LEACH-MH (Max Heap) [18] improves cluster head management. The number of nodes under a particular cluster is adjusted so that no cluster head is over loaded and load balancing is achieved.

Year 2013

- I-LEACH [19] improves network lifetime through improved inner network communications. Sensor nodes with higher residual energy, more neighbours, and shorter distance from the BS are selected as $\mathrm{CH}$ nodes.

- K-LEACH (K-medoids) [20] improves the network lifespan. It provide uniform clustering through the use of the k-medoids clustering algorithms.

- Q-LEACH (Quadrant)[21] improves overall energy consumption through the use of quadrant based directional routing. Nodes, which are near the sink node, may forward their messages. It combines the advantages of location and hierarchical routing protocols.

- LEACH-P (Pseudo Cluster Head) [22] improves the network load. Load monitor and load leisure mechanisms are used to control the load and stability of the network topology. When the cluster head node is limited in energy, a pseudo cluster head node replaced the cluster head node to keep the network alive.
- MODLEACH (Modified) [23] improves the network lifetime and throughput. It introduces efficient cluster head replacement and dual transmitting power levels. Hard and Soft thresholds are applied in the algorithms for better performance.

Year 2012

- N-LEACH [24] improves on the cluster head selection method, balances the energy consumption of sensor nodes and improves the network lifetime.

- EEE-LEACH (Energy Efficient Extended) [25] improves communication distance between nodes and introduces master cluster heads along with cluster heads.

- MG-LEACH (Multi Group) [26] improves network lifetime through consideration of redundancy of deployed nodes.

- Cell LEACH [27] improves the network lifetime through the use of clustering and celling such that once the clusters and cells are formed, they remain static in the network. Only the cells heads and cluster heads are changed afterwards.

- WLEACH (Wise) [28] improves CH selection by considering residual energy at the time of selection. Multi jump routing amongst nodes and sleep wakeup awareness are included.

- U-LEACH (Uniform [29] improves network lifetime through uniform distribution technique for $\mathrm{CH}$ selection such that all sensor nodes remain inside the transmission range of $\mathrm{CHs}$ and no nodes are left without being assigned to a cluster.

Year 2011

- B4-LEACH (Four Bridge) [5] improves the $\mathrm{CH}$ workload by introducing bridge nodes. $\mathrm{CH}$ nodes forward data to bridge nodes instead of $\mathrm{CH}$ nodes of other clusters.

- FZ-LEACH (Far Zone) [30] improves lifetime of WSN by addressing the shortcomings of with very large and very small clusters imposed by LEACH through the use of far zone sensor nodes.

- LEACH-SM (Spare Node Management) [31 improves the network lifetime through the introduction of spare nodes. The protocol provides an optimal spare selection and energy saving management of the spare nodes. The protocol reduces transmission of redundant packets to the $\mathrm{CH}$.

Year 2010

- LEACH-L [32] improves the communication between the $\mathrm{CH}$ and the BS. When the $\mathrm{CH}$ nodes are close to the BS, they communicate directly with the latter. When the $\mathrm{CH}$ nodes are far from the BS multi-hop communication is favoured.

- LEACH-B (Balanced) [33] improves on the number of $\mathrm{CH}$ nodes. For each round, after the selection of cluster heads, a second selection round is introduced to update the number of cluster heads based on the residual energy, consequently balancing the number of $\mathrm{CH}$ nodes.

- LEACH-D [34] improves $\mathrm{CH}$ nodes selection and network lifetime. It combines the idea of adjusting threshold functions of the nodes, fixed radius of clustering and multi-hop mechanism. 
- W-LEACH (Weighted) [35] improves network lifetime and average lifetime for sensors for uniform and non-uniform WSNs through improved aggregation of sensors' data.

- MR-LEACH (Multi-hop Routing) [36] improves network lifetime through network partitioning into different layers of clusters. $\mathrm{CH}$ nodes in adjacent layers collaborate to transmit data to the BS. Ordinary nodes join the clusters based on received signal strength indicators.

Year 2009

- LEACH-H (Hybrid) [37 improves the network lifetime by employing the simulated annealing algorithm to select $\mathrm{CH}$ nodes in the first round. The selected $\mathrm{CH}$ is then responsible for $\mathrm{CH}$ selection of its cluster in successive rounds for even distribution of $\mathrm{CH}$ nodes.

- T-LEACH (Threshold) [3] improves the network lifetime by minimising the number of cluster heads based on $\mathrm{CH}$ selection using the highest threshold of residual energy.

- V-LEACH (Vice) [6] improves the $\mathrm{CH}$ selection process. Besides having a $\mathrm{CH}$ in the cluster, there is a vice $\mathrm{CH}$ node that takes the role of the $\mathrm{CH}$ when the $\mathrm{CH}$ dies.

Year 2008

- LEACH-ME (Mobile Enhanced)[38] improves CH selection process based on mobility and residual energy constraint.

- MELEACH-L (More Energy Efficient) [39] improves channel assignment among neighbour clusters and cooperation among cluster head nodes in large WSN.

- Trust Based LEACH [40] improves security of routing while keeping the necessary functionalities of the LEACH protocol.

- TB-LEACH (Time Based) [41] improves system lifetime by providing a time based cluster head selection algorithm.

Year 2007

- M-LEACH (Multihop) [4] improves the communication within the network. It selects the optimal path between $\mathrm{CH}$ and BS through other cluster head nodes.

- E-LEACH (Energy) [4] improves the $\mathrm{CH}$ selection process. The algorithm makes us of rounds so that the nodes have same probability of becoming $\mathrm{CH}$ nodes in the first rounds. $\mathrm{CH}$ selection in subsequent rounds is done based on the residual energy of each node.

\section{Year 2006}

- LEACH-ET (Energy Threshold) [42] improves overhead of clustering. Cluster change occurs only when energy consumed by any $\mathrm{CH}$ nodes reach an energy threshold in a round. The protocols ensure that each node knows the energy threshold.

- LEACH-M (Mobile) [43] improves energy utilisation. TDMA scheduling is used to confirm whether a mobile node is in communication range with the $\mathrm{CH}$ or not. If not, the mobile node is removed from the member list the $\mathrm{CH}$.

Year 2005

- TL-LEACH (Two-Level) [44] improves energy utilisation by proposing primary and secondary levels cluster head selection.

Year 2002

- LEACH-C (Centralised) [45] improves the network lifetime and desired number of cluster generation. Clustering is centralised. The cluster heads are elected by the BS and evenly distributed among the nodes in the network.

Year 2000

- LEACH-F (Fixed) [2] improves CH selection overhead by using fixed clusters.

- LEACH [46] uses one hop. It is not suitable for large networks.

\subsection{Modifiers of LEACH with added concepts}

Next, the families of LEACH with added concepts and details on the improvements brought to LEACH are listed.

Year 2011

- HEEP [47] combines the cluster-based approach of LEACH and the chain based approached of PEGASIS, consequently referred to as the chains clustering protocol. It improves the energy dissipation in LEACH and the routing of PEGASIS.

- EADUC [48] improves energy consumption of nodes. The $\mathrm{CH}$ noded in EADUC can achieve balanced energy, good distribution and consistent coverage for all nodes.

Year 2005

- EECS [49] improves the distribution of $\mathrm{CH}$ nodes. It also allows dynamic sizing of the clusters based on the cluster distance from the BS.

Year 2004

- HEED [50] extends the LEACH protocol by considering the residual energy and node density for cluster selection.

Year 2002

- PEGASIS [7] introduces the chain-based concept. Each node communicates only with a close neighbour and takes turns transmitting to the BS instead of all nodes communicating directly to the BS.

Year 2001

- TEEN [10] aims at reducing energy spent on data transmission and is suitable for time critical data network. The algorithm makes us of a hard and a soft thresholds to control transmission.

3.2.1 Family of LEACH Modifiers. The modifiers of the different LEACH families and their corresponding features are given below.

Year 2016

- Improved EADUC (EADUC family) [51] improves upon the energy hole problem of multi-hop WSN through the use of non-uniform clustering. The protocol considers the location of the $\mathrm{BS}$, residual energy and number of neighbourhood nodes in the $\mathrm{CH}$ selection sub-phase. Clustering overhead is reduced by prolonging the per round data transmission phase. Major slots and mini slots are used for data collection. There is an overall increase in network lifetime. 
Year 2014

- R-HEED (Rotated) (HEED family) [52] improves network lifetime. It proposes a more energy efficient clustering phase than HEED.

\section{Year 2012}

- UHEED (Unequal) (HEED family) [53] improves network lifetime. It makes use of unequal clustering and balances the energy levels of cluster head nodes.

Year 2010

- H-HEED (Heterogeneous) (HEED family) [54] prolongs network lifetime. The protocol is for heterogeneous WSN, whereby cluster head selection is based on residual energy of nodes.

- EAPHRN (Energy Aware PEGASIS Based Hierarchical Routing) (PEGASIS family) [55] improves lifetime and throughput of the network. Instead of selecting the next closest node for chain construction it connects a random node that is located not farther than a distance threshold. The threshold is considered such that there is low rate of energy consumption.

- PDCH (PEGASIS with Double Cluster Head) (PEGASIS family) [56] improves network lifetime and balances the load of nodes. Long chaining and dynamic cluster formation are avoided.

- H-PEGASIS (PEGASIS family) [8] improves the data gathering problems and delay of packets transmission to the BS.

- Grid-PEGASIS - PEGASIS (PEGASIS family) [57] improves energy efficiency and balancing. It prevents the long hop caused by the greedy algorithm. The sensing area is divided into small grid areas.

- PEGASIS ANT (PEGASIS family) [58] improves optimisation and prolongs network lifetime. It uses ant colony algorithm instead of the greedy algorithm to construct the data chain.

Year 2006

- EB-PEGASIS (Energy Efficient) (PEGASIS family) [9] improves upon PEGASIS through the use of a distance threshold. It avoids long chains, thus enhancing energy consumption of nodes.

Year 2002

- H-PEGASIS (Hierarchical) (PEGASIS family) improves the transmission delay of PEGASIS by considering the energy and delay metrics. It provides hierarchical extension to PEGASIS with nodes chain forming a tree-like hierarchy.

- APTEEN (TEEN family) [11 combines all features of TEEN. It is adapted for proactive and reactive networks such that data are sent periodically and sudden attribute value changes are responded to.

\section{DISCUSSION OF LEACH}

The LEACH protocol since its inception is considered as the state of the art hierarchical clustering protocol. Different works and research on hierarchical clustering is still to this date considering the LEACH protocol in one-way or the other. The popularity of the protocol remains that the protocol provides a basic scenario and implementation of a standard clustering hierarchical protocol. Other improvements added to the protocol and introduction of different family with LEACH as their basis have all try to improve the protocol with the ultimate objectives being to prolong the network lifetime.

\section{CONCLUSION}

The limited energy of the nodes in a WSN affects the entire network. Energy efficiency is an area that attracts much research in the field of WSNs because of their wide applications in numerous areas. The LEACH protocol has long been considered as the basis of many other protocols. The LEACH protocol provides an agreeable working model of self-organisation and communication of nodes. However, it suffers from different drawbacks, the discussion and consideration of which have resulted in the culmination of numerous research outcomes and protocols. This paper has summarised some of these protocols and has highlighted the timeline and improvement brought to the LEACH protocol.

\section{REFERENCES}

[1] S. P. Singh and S. C. Sharma. A survey on cluster based routing protocols in wireless sensor networks. Procedia Computer Science, 45(9):687-695, 2015.

[2] W. Heinzelman. Application-Specific Protocol Architectures for Wireless Networks. PhD thesis, Massachusetts Institute of Technology, 2000.

[3] J. Hong, S. Lee J. Kook, D. Kwon, and S. Yi. T-LEACH: The method of threshold-based cluster head replacement for wireless sensor networks. Information Systems Frontiers, 11(5):513-521, 2009.

[4] F. Xiangning and S. Yulin. Improvement on LEACH protocol of wireless sensor network. In Proc. of International Conference on Sensor Technologies and Applications, pages 260-264, 2007.

[5] N. Laloo, M. S. Sunhaloo, and R. K. Subramanian. The b4-leach protocol for improved homogeneous wireless sensor network lifetime. In Proc. of the 2011 Third Congress in Applied Computing, Computer Science and Computer Engineering, volume 1, pages 99-104, 2011.

[6] M. Bani Yassein, A. Al-zou'bi, Y. Khamayseh, and W. Mardini. Improvement on LEACH protocol of wireless sensor network (VLEACH). International Journal of Digital Content Technology and its Applications, 3(2):182-186, 2009.

[7] S. Lindsey and C. S. Raghavendra. PEGASIS: Power-efficient gathering in sensor information systems. In Proc. of IEEE Aerospace Conference, volume 3, pages 1125-1130, 2002.

[8] S. K. Singh, M. P. Singh, and D. K. Singh. A survey of energy-efficient hierarchical cluster-based routing in wireless sensor networks. International Journal of Advanced Networking and Applications, 2(2):570-580, 2010.

[9] L. Yueyang, J. Hong, and Y. Guangxin. An energy-efficient PEGASIS-based enhanced algorithm in wireless sensor networks. China Communications, pages 91-97, 2006.

[10] A. Manjeshwar and D. P. Agrawal. TEEN: a routing protocol for enhanced efficiency in wireless sensor networks. In Proc. of 15th International Parallel and Distributed Processing Symposium, pages 2009-2015, 2001.

[11] A. Manjeshwar and D. P. Agrawal. A hybrid protocol for efficient routing and comprehensive information retrieval in wireless sensor networks. In Proc. of 16th International Parallel and Distributed Processing Symposium, 2002. 
[12] M. Madheswaran and R. N. Shanmugasundaram. Enhancements of LEACH algorithm for wireless networks: A review. Internation Journal On Communication Technology, 4(4):821-827, 2013.

[13] J. Gnanambigai, N. Rengarajan, and K. Anbukkarasi. LEACH and its descendant protocols: A survey. International Journal of Communication and Computer Technologies, 1(3):15-21, 2012.

[14] H. Dhawan and S. Waraich. A comparative study on leach routing protocol and its variants in wireless sensor networks: a survey. International Journal of Computer Applications, 95(8), 2014.

[15] N. V. Deshmukh and Y. M. Patil. Review of LEACH variants. International Journal of Advanced Research in Computer and Communication Engineering, 4(9), 2015.

[16] P. K. Batra and K. Kant. LEACH-MAC: a new cluster head selection algorithm for wireless sensor networks. Wireless Networks, 22(1):49-60, 2016.

[17] S. E. Khediri, A. Wei N. Nasri, and A. Kachouri . A new approach for clustering in wireless sensors networks based on LEACH. Procedia Computer Science, 32:1180-1185, 2014.

[18] R. Kamboj and R. Chahal. Enhanced LEACH for better cluster management using MAX-HEAP. International Journal of Computer Science and Information Technologies, 5(3):43314335, 2014.

[19] Z. Beiranvand, A. Patooghy, and M. Fazeli. I-LEACH: An efficient routing algorithm to improve performance $\&$ to reduce energy consumption in wireless sensor networks. In Proc. of 5th Conference on Information and Knowledge Technology, pages 13-18, 2013.

[20] P. Bakaraniya and S. Mehta. K-LEACH: An improved LEACH protocol for lifetime improvement in wsn. International Journal of Engineering Trends and Technology, 4(5):1521-1526, 2013.

[21] K. Anbukkarasi and J. Gnanambigai. An energy efficient quadrant based clustering approach for wireless sensor networks. In Proc. of Int. Conf. on Emerging Trends in Engineering and Technology, pages 269-277, 2013.

[22] C. Leng, H. Yu, J. Wang, and J. Huang. Securing personal health records in clouds by enforcing sticky policies. TELKOMNIKA, 11(4):2200-2208, 2013.

[23] D. Mahmood, N. Javaid, S. Mahmood, S. Qureshi, A. M. Memon, and T. Zaman. Modleach: a variant of leach for wsns. In Proc. of 2013 Eighth International Conference on Broadband, Wireless Computing, Communication and Applications, pages 158-163, 2013.

[24] R. K. Tripathi, Y. N. Singh, and N. K. Verma. N-LEACH, a balanced cost cluster-heads selection algorithm for wireless sensor network. In Proc. of 2012 National Conference on Communications, pages 1-5, 2012.

[25] M. Sharma and K. Sharma. An energy efficient extended LEACH (eee leach). In Proc. of 2012 International Conference on Communication Systems and Network Technologies, pages 377-382, 2012.

[26] M. Haneef, Z. Wenxun, and Z. Deng. MG-LEACH: Multi group based LEACH an energy efficient routing algorithm for wireless sensor network. In Proc. of 2012 14th International Conference on Advanced Communication Technology, pages 179-183, 2012.
[27] A. Yektaparast, F.-H. Nabavi, and A. Sarmast. An improvement on LEACH protocol (Cell-LEACH). In Proc. of 2012 14th International Conference on Advanced Communication Technology, pages 992-996, 2012.

[28] C. Yueyun, Z. Yue-Long, A. Jianwei, and L. Qian. An energysaving routing protocol based on LEACH. In Proc. of 2012 Fourth International Conference on Multimedia Information Networking and Security, pages 608-611, 2012.

[29] N. Majadi. U-LEACH: A routing protocol for prolonging lifetime of wireless sensor networks. International Journal of Engineering Research and Applications, 2(4):1649-1652, 2012.

[30] V. Katiyar, N. Chand, G. C. Gautam, and A. Kumar. Improvement in LEACH protocol for large-scale wireless sensor networks. In Proc. of 2011 International Conference on Emerging Trends in Electrical and Computer Technology, pages 1070-1075, 2011.

[31] B. A. Bakr and L. Lilien. LEACH-SM: A protocol for extending wireless sensor network lifetime by management of spare nodes. In Proc. of 2011 International Symposium on Modeling and Optimization in Mobile, Ad Hoc and Wireless Networks, page 375, 2011.

[32] F. Shang and Y. Lei. An energy-balanced clustering routing algorithm for wireless sensor network. Wireless Sensor Network, 2(10):777-785, 2010.

[33] M. Tong and M. Tang. LEACH-B: An improved LEACH protocol for wireless sensor network. In Proc. of 2010 6th International Conference on Wireless Communications Networking and Mobile Computing, pages 1-4, 2010.

[34] Y. Liu, K. Xu, Z. Luo, and L. Chen. A reliable clustering algorithm base on LEACH protocol in wireless mobile sensor networks. In Proc. of 2010 2nd International Conference on Mechanical and Electrical Technology, pages 692-696, 2010.

[35] H. M. Abdulsalam and L. K. Kamel. W-leach: Weighted low energy adaptive clustering hierarchy aggregation algorithm for data streams in wireless sensor networks. In Proc. of 2010 IEEE International Conference on Data Mining Workshops, pages $1-8,2010$

[36] M. O. Farooq, A. B. Dogar, and G. A. Shah. MR-LEACH: Multi-hop routing with low energy adaptive clustering hierarchy. In Proc. of 2010 Fourth International Conference on Sensor Technologies and Applications, pages 262-268, 2010.

[37] W. Wang, Q. Wang, W. Luo, M. Sheng, W. Wu, and L. Hao. Leach-H: An improved routing protocol for collaborative sensing networks. In Proc. of International Conference on Wireless Communications \& Signal Processing, 2009, pages $1-5,2009$.

[38] G. S. Kumar, M. V. V. Paul, G. Athithan, and K. P. Jacob. Routing protocol enhancement for handling node mobility in wireless sensor networks. In Proc. of 2008 IEEE Region 10 Conference TENCON 2008, pages 1-6, 2008.

[39] J. Chen and H. Shen. MELEACH-L: More energy-efficient LEACH for large-scale WSNs. In Proc. of 4th International Conference on Wireless Communications, Networking and Mobile Computing, pages 1-4, 2008.

[40] Fei Song and Baohua Zhao. Trust-based LEACH protocol for wireless sensor networks. In Proc. of Second International Conference on Future Generation Communication and Networking, volume 1, pages 202-207, 2008. 
[41] H. Junping, J. Yuhui, and D. Liang. A time-based cluster-head selection algorithm for LEACH. In Proc. of IEEE Symposium on Computers and Communications, pages 1172-1176, 2008.

[42] L. Lijun, W. Hongtao, and C. Peng. Discuss in round rotation policy of hierarchical route in wireless sensor networks. In Proc. of International Conference on Wireless Communications, Networking and Mobile Computing, pages 1-5, 2006.

[43] D.-S. Kim and Y. J. Chung. Self-organization routing protocol supporting mobile nodes for wireless sensor network. In Proc. of First International Multi-Symposiums on Computer and Computational Sciences, volume 2, pages 622-626, 2006.

[44] V. Loscri, G. Morabito, and S. Marano. A two-levels hierarchy for low-energy adaptive clustering hierarchy (TLLEACH). In Proc. of IEEE 62nd Vehicular Technology Conference, volume 62, pages 1809-1813, 2005.

[45] W. B. Heinzelman, A. P. Chandrakasan, and H. Balakrishnan. An application-specific protocol architecture for wireless microsensor networks. IEEE Transactions on Wireless Coтmunications, 1(4):660-670, 2002.

[46] W. B. Heinzelman, A. Chandrakasan, and H. Balakrishnan. Energy-efficient communication protocol for wireless microsensor networks. In Proc. of Proceedings of the Hawaii international conference on System sciences, pages 1-10, 2000.

[47] D. E. Boubiche and A. Bilami. HEEP (Hybrid Energy Efficiency Protocol) based on chain clustering. International Journal of Sensor Networks, 10(1/2):25-35, 2011.

[48] J. Yu, Y. Qi, G. Wang, Q. Guo, and X. Gu. An energy-aware distributed unequal clustering protocol for wireless sensor networks. International Journal of Distributed Sensor Networks, 2011.

[49] Mao Ye, Chengfa Li, Guihai Chen, and Jie Wu. EECS: An energy efficient clustering scheme in wireless sensor networks. In Proc. of 24th IEEE International Performance Computing and Communications Conference, pages 535-540, 2005.

[50] O. Younis and S. Fahmy. HEED: A hybrid, energy-efficient, distributed clustering approach for ad-hoc sensor networks. IEEE Transactions on Mobile Computing, 3(4):366-379, 2004.

[51] V. Gupta and R. Pandey. An improved energy aware distributed unequal clustering protocol for heterogeneous wireless sensor networks. Engineering Science and Technology, an International Journal, 19(2):1050-1058, 2016.

[52] W. Mardini, M. B. Yassein, Y. Khamayseh, and B. A. Ghaleb. Rotated hybrid, energy-efficient and distributed (R-HEED) clustering protocol in WSN. WSEAS Transactions on Communications, 13:275-290, 2014.

[53] E. Ever, R. Luchmun, L. Mostarda, A. Navarra, and P. Shah. UHEED - an unequal clustering algorithm for wireless sensor networks. In Proc. of 1st International conference on sensor networks, Italy, pages 185-193, 2012.

[54] H. Kour and A. K. Sharma. Hybrid energy efficient distributed protocol for heterogeneous wireless sensor network. International Journal of Computer Applications, 4(6):1-5, 2010.

[55] H. Al-Hasan, M. Qatawneh, A. Sleit, and W. Almobaideen. EAPHRN: Energy-aware PEGASIS-based hierarchal routing protocol for wireless sensor networks. Journal of American Science, 7(8), 2011.

[56] W. Linping, B. Wu, C. Zhen, and W. Zufeng. Improved algorithm of PEGASIS protocol introducing double cluster heads in wireless sensor network. In Proc. of 2010 International Conference on Computer, Mechatronics, Control and Electronic Engineering, volume 1, pages 148-151, 2010.

[57] Y.-F. Huang, C.-C. Chen, N.-C. Wang, J.-Y. Lin, and C.-M. Chen. Performance of a data gather scheme with novel chain construction for wireless sensor networks. In Proc. of the 6th International Wireless Communications and Mobile Computing Conference, pages 1208-1212, 2010.

[58] W. Guo, W. Zhang, and G. Lu. PEGASIS protocol in wireless sensor network based on an improved ant colony algorithm. In Proc. of 2010 Second International Workshop on Education Technology and Computer Science, volume 3, pages 6467, 2010 